\title{
Co-Finiteness and Co-Emptiness of Reachability Sets in Vector Addition Systems with States ${ }^{\star}$
}

\author{
Petr Jančar ${ }^{1}$, Jérôme Leroux ${ }^{2}$, and Grégoire Sutre ${ }^{2}$ \\ 1 Dept of Comp. Sci., Faculty of Science, Palacký Univ. Olomouc, Czech Rep. \\ petr.jancar@upol.cz \\ 2 Univ. Bordeaux \& CNRS, LaBRI, UMR 5800, Talence, France \\ jerome.leroux@labri.fr, gregoire.sutre@labri.fr
}

\begin{abstract}
The coverability and boundedness problems are well-known exponential-space complete problems for vector addition systems with states (or Petri nets). The boundedness problem asks if the reachability set (for a given initial configuration) is finite. Here we consider a dual problem, the co-finiteness problem that asks if the complement of the reachability set is finite; by restricting the question we get the coemptiness (or universality) problem that asks if all configurations are reachable.

We show that both the co-finiteness problem and the co-emptiness problem are complete for exponential space. While the lower bounds are obtained by a straightforward reduction from coverability, getting the upper bounds is more involved; in particular we use the bounds derived for reversible reachability by Leroux in 2013.

The studied problems have been motivated by a recent result for structural liveness of Petri nets; this problem has been shown decidable by Jančar in 2017 but its complexity has not been clarified. The problem is tightly related to a generalization of the co-emptiness problem for non-singleton sets of initial markings, in particular for downward closed sets. We formulate the problems generally for semilinear sets of initial markings, and in this case we show that the co-emptiness problem is decidable (without giving an upper complexity bound) and we formulate a conjecture under which the co-finiteness problem is also decidable.
\end{abstract}

\section{Introduction}

Context. Analysis of behavioural properties of (models of) systems is a natural and wide area of study; the decidability and complexity questions for respective properties are an important part of such research. As the most relevant for us we recall the reachability and liveness problems for Petri nets.

A concrete source of motivation for us has been the recent paper [7] that answered the decidability question for structural liveness in Petri nets positively;

\footnotetext{
* This work was supported by the grant GAČR:18-11193S of the Czech Grant Agency (P. Jančar) and by the grant ANR-17-CE40-0028 of the French National Research Agency ANR, project BraVAS (J. Leroux and G. Sutre)
} 
the open status of this question was previously recalled, e.g., in [2]. It is natural to continue with studying the computational complexity of this problem. Here we contribute indirectly to this topic by studying some related naturally arising problems concerning reachability sets.

The algorithm in [7] reduces the structural liveness problem to the question if a Petri net with a downward closed set of initial markings is "universal", in the sense that every marking is reachable from the initial ones. This question has been solved by using the involved result proved in [8], namely that there is an algorithm that halts with a Presburger description of the reachability set when this set is semilinear. Since this approach is not constructive, it does not provide any complexity upper bound. This led us to consider the universality problem, which we call the co-emptiness problem, on its own. There is also a naturally related co-finiteness problem asking if a set of initial markings allows to reach all but finitely many markings; this problem can be thus seen as dual to the well-known boundedness problem that asks if the reachability set is finite.

Contributions. We formulate the co-emptiness and co-finiteness problem generally for semilinear sets of initial markings. We show that the co-emptiness problem is decidable using a reduction to [8] that is similar to the above-mentioned approach used in [7] to decide the structural liveness problem. As before, no complexity upper bound can be derived from that approach. In the case of the co-finiteness problem we are even not sure with decidability, but we formulate a conjecture under which the problem is decidable.

We then consider restrictions to the case with finite sets of initial markings and then in particular to the case with singleton sets of initial markings.

In the case of finite initial sets we show that the co-emptiness problem reduces in logarithmic space to the reachability problem. The converse reduction (reachability to co-emptiness) is left open.

In the case of singleton initial sets we show EXPSPACE-completeness for both co-emptiness and co-finiteness. This is the main technical result of the paper. While the lower bound is obtained by an easy reduction from the coverability problem (a well known EXPSPACE-complete problem, similarly as boundedness), getting the upper bound is more involved. Using the bound obtained for reversible reachability by Leroux in [9], we reduce the co-emptiness problem (with a single initial marking) to a large number of coverability questions in a large Petri net. The latter is bounded in such a way that the questions can be still answered in exponential space, using Rackoff's technique [13].

Though our results do not improve our knowledge about the complexity of structural liveness directly, we show that a related problem, namely the structural deadlock-freedom problem is tightly related (interreducible in polynomial time) with the co-emptiness problem in the case of downward closed sets of initial markings.

We have found more convenient to present our results on the model of vector addition systems with states, or shortly VASSs. This model is equivalent to Petri nets and all our results, while proved for VASSs, also hold for Petri nets. 
Outline. In Section 2 we recall some preliminary notions, such as vector addition systems with states and semilinear sets. Section 3 defines the co-emptiness problem and the co-finiteness problem, and presents our partial decidability results for the general case and for the restriction to finite sets of initial configurations. The main result is contained in Section 4 where we show the EXPSPACEcompleteness of co-emptiness and co-finiteness in the case with singleton sets of initial configurations. Section 5 presents two applications of the co-emptiness problem: we recall the structural liveness, and show the tight relation of structural deadlock-freedom to the co-emptiness problem with downward closed sets of initial configurations. We conclude the paper by Section 6 .

\section{Preliminaries}

By $\mathbb{Z}$ we denote the set of integers, and by $\mathbb{N}$ the set $\{0,1,2, \ldots\}$ of nonnegative integers. By $[i, j]$, where $i, j \in \mathbb{Z}$, we denote the set $\{i, i+1, \ldots, j\}$ (which is empty when $i>j$ ).

For a vector $v \in \mathbb{Z}^{d}(d \in \mathbb{N})$, by $v(i)$ we denote the $i$-th component of $v$. On $\mathbb{Z}^{d}$ we define the operations,+- and the relations $\geq, \leq$ componentwise. For $v_{1}, v_{2} \in \mathbb{Z}^{d}$ we thus have $v_{1}+v_{2}=w$ where $w(i)=v_{1}(i)+v_{2}(i)$ for all $i \in[1, d]$; we have $v_{1} \leq v_{2}$ iff $v_{1}(i) \leq v_{2}(i)$ for all $i \in[1, d]$. For $k \in \mathbb{N}$ and $v \in \mathbb{Z}^{d}$ we put $k \cdot v=(k \cdot v(1), k \cdot v(2), \cdots, k \cdot v(d))$; we also write $k v$ instead of $k \cdot v$.

Slightly abusing notation, by $\left(v_{1}, v_{2}, \ldots, v_{m}\right)$ where $v_{i} \in \mathbb{Z}^{d_{i}}$ for $i \in[1, m]$ we do not denote an $m$-tuple of vectors but the corresponding vector of dimension $d=\sum_{i \in[1, m]} d_{i}$.

The norm $\|v\|$ of a vector $v \in \mathbb{Z}^{d}$ is $\max \{|v(i)| ; i \in[1, d]\}$, and the norm $\|V\|$ of a finite set $V \subseteq \mathbb{Z}^{d}$ is $\max \{\|v\| ; v \in V\}$; here we stipulate $\max \emptyset=0$.

When the dimension $d$ is clear from context, by $\mathbf{0}$ we denote the zero vector $(\mathbf{0}(i)=0$ for all $i \in[1, d])$, and by $\mathbf{e}_{i}(i \in[1, d])$ the vector satisfying $\mathbf{e}_{i}(i)=1$ and $\mathbf{e}_{i}(j)=0$ for all $j \in[1, d] \backslash\{i\}$.

For a set $A$, by $A^{*}$ we denote the set of finite sequences of elements of $A$, and by $\varepsilon$ we denote the empty sequence. For $w \in A^{*},|w|$ denotes its length.

Vector addition systems with states (VASSs). A vector addition system with states (a VASS) is a tuple $\mathcal{V}=(d, Q, \mathcal{A}, T)$ where $d \in \mathbb{N}$ is the dimension, $Q$ is the finite set of (control) states, $\mathcal{A} \subseteq \mathbb{Z}^{d}$ is the finite set of actions, and $T \subseteq Q \times \mathcal{A} \times Q$ is the finite set of transitions. We often present $t \in T$ where $t=\left(q, \mathbf{a}, q^{\prime}\right)$ as $q \stackrel{\mathbf{a}}{\rightarrow} q^{\prime}$ or $t: q \stackrel{\mathbf{a}}{\rightarrow} q^{\prime}$.

The set of configurations of $\mathcal{V}=(d, Q, \mathcal{A}, T)$ is the set $Q \times \mathbb{N}^{d}$; we rather present a configuration $(q, v)$ as $q(v)$ (where $q \in Q, v \in \mathbb{N}^{d}$ ). For actions $\mathbf{a} \in \mathcal{A}$ we define relations $\stackrel{\mathrm{a}}{\rightarrow} \mathcal{V}$ on the set $Q \times \mathbb{N}^{d}$ of configurations by putting

$$
q(v) \stackrel{\mathbf{a}}{\rightarrow}_{\mathcal{V}} q^{\prime}\left(v^{\prime}\right) \text { if } q \stackrel{\mathbf{a}}{\rightarrow} q^{\prime} \text { is a transition in } T \text { and } v^{\prime}=v+\mathbf{a} .
$$

Hence for a transition $q \stackrel{\text { a }}{\rightarrow} q^{\prime}$ and $v \in \mathbb{N}^{d}$ we have $q(v) \stackrel{\text { a }}{\rightarrow} \mathcal{V} q^{\prime}(v+\mathbf{a})$ iff $v+\mathbf{a} \geq \mathbf{0}$. 
Relations $\stackrel{\text { a }}{\rightarrow} \mathcal{V}$ are naturally extended to relations $\stackrel{\alpha}{\rightarrow} \mathcal{V}$ for $\alpha \in \mathcal{A}^{*}$; we write just $\stackrel{\alpha}{\rightarrow}$ instead of $\stackrel{\alpha}{\rightarrow} \mathcal{V}$ when $\mathcal{V}$ is clear from context. The extension is defined inductively: we put $q(v) \stackrel{\varepsilon}{\rightarrow} q(v)$; if $q(v) \stackrel{\text { a }}{\rightarrow} q^{\prime}\left(v^{\prime}\right)$ and $q^{\prime}\left(v^{\prime}\right) \stackrel{\alpha}{\rightarrow} q^{\prime \prime}\left(v^{\prime \prime}\right)$, then $q(v) \stackrel{\mathbf{a} \alpha}{\longrightarrow} q^{\prime \prime}\left(v^{\prime \prime}\right)$. We note that $q(v) \stackrel{\alpha}{\rightarrow} q^{\prime}\left(v^{\prime}\right)$ where $\alpha=\mathbf{a}_{1} \mathbf{a}_{2} \cdots \mathbf{a}_{m}$ implies that $v^{\prime}=v+\sum_{i \in[1, m]} \mathbf{a}_{i}$. We also note the monotonicity:

$$
\text { if } q(v) \stackrel{\alpha}{\rightarrow} q^{\prime}\left(v^{\prime}\right) \text {, then for any } \bar{v} \geq v \text { we have } q(\bar{v}) \stackrel{\alpha}{\rightarrow} q^{\prime}\left(v^{\prime}+\bar{v}-v\right) \text {. }
$$

Reachability sets. Given a VASS $\mathcal{V}=(d, Q, \mathcal{A}, T)$, by $q(v) \stackrel{*}{\rightarrow} \mathcal{V} q^{\prime}\left(v^{\prime}\right)$, or by $q(v) \stackrel{*}{\rightarrow} q^{\prime}\left(v^{\prime}\right)$ when $\mathcal{V}$ is clear from context, we denote that $q^{\prime}\left(v^{\prime}\right)$ is reachable from $q(v)$, i.e., that $q(v) \stackrel{\alpha}{\rightarrow} q^{\prime}\left(v^{\prime}\right)$ for some $\alpha \in \mathcal{A}^{*}$. The reachability set for an (initial) configuration $q(v)$ is the set

$$
[q(v)\rangle_{\mathcal{V}}=\left\{q^{\prime}\left(v^{\prime}\right) \mid q(v) \stackrel{*}{\rightarrow} \mathcal{V} q^{\prime}\left(v^{\prime}\right)\right\} .
$$

For a set $C \subseteq Q \times \mathbb{N}^{d}$ of (initial) configurations we put

$$
[C\rangle_{\mathcal{V}}=\bigcup_{q(v) \in C}[q(v)\rangle_{\mathcal{V}} .
$$

We also write just $[q(v)\rangle$ and $[C\rangle$ when $\mathcal{V}$ is clear from context.

We write $q(v) \stackrel{*}{\rightarrow} C$ if there is $q^{\prime}\left(v^{\prime}\right) \in C$ such that $q(v) \stackrel{*}{\rightarrow} q^{\prime}\left(v^{\prime}\right)$; similarly $C \stackrel{*}{\rightarrow} q(v)$ if there is $q^{\prime}\left(v^{\prime}\right) \in C$ such that $q^{\prime}\left(v^{\prime}\right) \stackrel{*}{\rightarrow} q(v)$.

Semilinear sets of configurations. A set $C \subseteq Q \times \mathbb{N}^{d}$ is linear if

$$
C=\left\{q\left(b+n_{1} p_{1}+\cdots+n_{k} p_{k}\right) \mid n_{1}, \ldots, n_{k} \in \mathbb{N}\right\}
$$

for some $q \in Q, k \in \mathbb{N}$, and $b, p_{1}, \ldots, p_{k} \in \mathbb{N}^{d}$. A set $C \subseteq Q \times \mathbb{N}^{d}$ is semilinear if $C=L_{1} \cup L_{2} \cup \cdots \cup L_{m}$ for some $m \in \mathbb{N}$ and linear sets $L_{j}, j \in[1, m]$.

We recall that semilinear sets correspond to the sets definable in Presburger arithmetic [4].

Vector addition systems (VASs). A vector addition system (VAS) is a VASS $(d, Q, \mathcal{A}, T)$ where $Q$ is a singleton. In this case the single control state plays no role, in fact; it is thus natural to view a VAS as a pair $\mathcal{U}=(d, \mathcal{A})$ for a finite set $\mathcal{A} \subseteq \mathbb{Z}^{d}$. The configurations are here simply $v \in \mathbb{N}^{d}$, and for $\mathbf{a} \in \mathcal{A}$ we have

$$
v \stackrel{\mathbf{a}}{\rightarrow} v^{\prime} \text { iff } v^{\prime}=v+\mathbf{a}\left(\text { for any } v, v^{\prime} \in \mathbb{N}^{d}\right) \text {. }
$$

We write $[v\rangle_{\mathcal{U}}$, or just $[v\rangle$, for the reachability set of $v$. For a VAS the terms "action" and "transition" are identified.

Binary and unary presentations. Instances of the problems that we will consider comprise VASSs and (presentations of semilinear sets of) configurations. We implicitly assume that the numbers in the respective vectors are presented in binary. When giving a complexity lower bound, we will explicitly refer to a unary presentation to stress the substance of the lower bound. 


\section{Co-Finiteness and Co-Emptiness of Reachability Sets}

Now we introduce the two main problems considered in this paper.

Co-finiteness. The co-finiteness problem:

Instance: a $\operatorname{VASS} \mathcal{V}=(d, Q, \mathcal{A}, T)$ and

a (presentation of a) semilinear set $C \subseteq Q \times \mathbb{N}^{d}$.

Question: is $[C\rangle$ co-finite, i.e., is the set $\left(Q \times \mathbb{N}^{\bar{d}}\right) \backslash[C\rangle$ finite ?

Co-emptiness (or universality). By narrowing the co-finiteness question we get the co-emptiness problem:

Instance: a $\operatorname{VASS} \mathcal{V}=(d, Q, \mathcal{A}, T)$ and

a (presentation of a) semilinear set $C \subseteq Q \times \mathbb{N}^{d}$.

Question: is $[C\rangle$ co-empty, i.e., is $[C\rangle=Q \times \mathbb{N}^{d}$ ?

We note that co-emptiness can be also naturally called universality.

\subsection{Decidability of the General Problems}

We recall the classical reachability problem, defined as follows:

Instance: a VASS $\mathcal{V}$ and two configurations $q(v), q^{\prime}\left(v^{\prime}\right)$.

Question: is $q(v) \stackrel{*}{\rightarrow} \mathcal{V} q^{\prime}\left(v^{\prime}\right)$ ?

The problem is decidable [12] but its complexity remains elusive; the problem is known to be EXPSPACE-hard [11], and the best known upper bound is nonprimitive recursive [10].

By adding the acceleration techniques in [8], and decidability of Presburger arithmetic, it is straightforward to derive decidability of the co-emptiness problem. We first recall a crucial fact.

Theorem 1 (reformulation of Lemma XI.1 of [8]). Given a VASS $\mathcal{V}=$ $(d, Q, \mathcal{A}, T)$ and a semilinear set $C$ of configurations, for every semilinear set $D \subseteq[C\rangle_{\mathcal{V}}$ there is a sequence $\alpha_{1}, \ldots, \alpha_{k}$ of words in $\mathcal{A}^{*}$ such that for every $q(v) \in D$ we have

$$
C \stackrel{\alpha_{1}^{n_{1}} \ldots \alpha_{k}^{n_{k}}}{\longrightarrow} \mathcal{V} q(v)
$$

for some $n_{1}, \ldots, n_{k} \in \mathbb{N}$.

We thus deduce that the co-emptiness problem can be decided by the following two procedures that are executed concurrently:

- One procedure systematically searches for some configuration $q(v)$ such that $q(v) \notin[C\rangle$ which is verified by using an algorithm deciding (non)reachability; this search succeeds iff $[C\rangle$ is not co-empty. 
- The other procedure systematically searches for some words $\alpha_{1}, \ldots, \alpha_{k}$ such that for every configuration $q(v)$ there are $n_{1}, \ldots, n_{k}$ in $\mathbb{N}$ such that $C \stackrel{\alpha_{1}^{n_{1}} \ldots \alpha_{k}^{n_{k}}}{\longrightarrow} q(v)$. This property (of $\alpha_{1}, \ldots, \alpha_{k}$ ) can be formulated in Presburger arithmetic and is thus decidable. The search succeeds iff $[C\rangle$ is coempty.

However, the complexity of the co-emptiness problem is still open. In fact, we even have no reduction from or to the reachability problem.

The decidability status of the co-finiteness problem is not clear. We show how to solve the problem under a conjecture. Let us first introduce the notion of inductive set. A set of configurations $D$ of a $\operatorname{VASS} \mathcal{V}=(d, Q, \mathcal{A}, T)$ is inductive if for every configuration $q(v)$ in $D$ and every transition $t: q \stackrel{\text { a }}{\rightarrow} q^{\prime}$ in $T$ such that $v+\mathbf{a} \geq \mathbf{0}$, we have $q^{\prime}(v+\mathbf{a}) \in D$. We observe that if an inductive set $D$ contains a set of initial configurations $C$ then $[C\rangle \subseteq D$. Moreover, we can effectively decide if a semilinear set $D$ is inductive. We introduce the following conjecture.

Conjecture 2. Given a VASS $\mathcal{V}$ and a semilinear set $C$ of configurations, if $[C\rangle_{\mathcal{V}}$ is co-infinite (i.e., not co-finite), then there is an inductive semilinear set $D$ such that $C \subseteq D$ (hence also $[C\rangle_{\mathcal{V}} \subseteq D$ ) and $D$ is co-infinite.

Under that conjecture, the co-finiteness problem can be also decided by two algorithmic procedures executed concurrently:

- One procedure systematically searches for some inductive co-infinite semilinear set $D$ that contains $C$; this search succeeds iff $[C\rangle$ is co-infinite (under the conjecture).

- The other procedure systematically searches for some words $\alpha_{1}, \ldots, \alpha_{k}$ and a natural number $n$, such that for every configuration $q(v)$ with $\|v\| \geq n$ there are $n_{1}, \ldots, n_{k}$ in $\mathbb{N}$ satisfying $C \stackrel{\alpha_{1}^{n_{1}} \ldots \alpha_{k}^{n_{k}}}{\longrightarrow} q(v)$. This property (of $\alpha_{1}, \ldots, \alpha_{k}$ and $n$ ) can be formulated in Presburger arithmetic, and is thus decidable. The search succeeds iff $[C\rangle$ is co-finite thanks to Theorem 1. Indeed, when the reachability set $[C\rangle$ is co-finite then it is semilinear, and we can apply Theorem 1 with $D=[C\rangle$.

Hence we have derived:

Theorem 3. The co-emptiness problem is decidable.

The co-finiteness problem is decidable when assuming validity of Conjecture 2.

\subsection{Finitely Many Initial Configurations}

As already mentioned, we have no complexity upper bound for the (decidable) co-emptiness problem in our general form. In the rest of this section we focus on the FMIC co-emptiness problem, and the FMIC co-finiteness problem, where "FMIC" refers to "Finitely Many Initial Configurations". We give the result captured by Theorem 5 . 
Lemma 4. Given a VASS $\mathcal{V}=(d, Q, \mathcal{A}, T)$ and any set $C \subseteq Q \times \mathbb{N}^{d}$, we have

$$
[C\rangle=Q \times \mathbb{N}^{d} \text { iff }[C\rangle \supseteq D_{1} \cup D_{2}
$$

where $D_{1}=\left\{q\left(v+\mathbf{e}_{i}\right) \mid q(v) \in C, i \in[1, d]\right\}$ and $D_{2}=\{q(\boldsymbol{O}) \mid q \in Q\}$.

Proof. If $[C\rangle=Q \times \mathbb{N}^{d}$, then we trivially have $[C\rangle \supseteq D_{1} \cup D_{2}$.

Let us now assume $[C\rangle \supseteq D_{1}$. We show that

$$
q(v) \in[C\rangle \text { implies } q\left(v+\mathbf{e}_{i}\right) \in[C\rangle
$$

(for all $q \in Q, v \in \mathbb{N}^{d}, i \in[1, d]$ ). Indeed, if $q_{0}\left(v_{0}\right) \stackrel{*}{\rightarrow} q(v)$ for some $q_{0}\left(v_{0}\right) \in C$, then $[C\rangle \ni q_{0}\left(v_{0}+\mathbf{e}_{i}\right)$ since $[C\rangle \supseteq D_{1}$ and $q_{0}\left(v_{0}+\mathbf{e}_{i}\right) \stackrel{*}{\rightarrow} q\left(v+\mathbf{e}_{i}\right)$ by monotonicity; hence $q\left(v+\mathbf{e}_{i}\right) \in[C\rangle$.

If, moreover, $[C\rangle \supseteq D_{2}$, then by (1) we get $q(v) \in[C\rangle$ for all $q \in Q, v \in \mathbb{N}^{d}$.

Theorem 5. The FMIC co-emptiness problem is logspace reducible to the reachability problem.

Proof. Given a VASS $\mathcal{V}=(d, Q, \mathcal{A}, T)$ and a finite set $C \subseteq Q \times \mathbb{N}^{d}$, deciding if $[C\rangle=Q \times \mathbb{N}^{d}$ boils down to verifying if each configuration in the finite set

$$
D_{1} \cup D_{2}=\left\{q_{1}\left(v_{1}\right), \ldots, q_{k}\left(v_{k}\right)\right\}
$$

defined in Lemma 4 is reachable from (a configuration in) $C$.

Let $\mathcal{V}^{\prime}$ arise from $\mathcal{V}$ by adding a fresh control state $q_{0}$ and transitions $q_{0} \stackrel{v}{\rightarrow} q$ for all $q(v) \in C$. Hence

$$
[C\rangle_{\mathcal{V}} \supseteq D_{1} \cup D_{2} \text { iff } q_{0}(\mathbf{0}) \stackrel{*}{\rightarrow} \mathcal{V}^{\prime} q_{j}\left(v_{j}\right) \text { for all } j \in[1, k] .
$$

Let us now consider a VASS $\mathcal{V}^{\prime \prime}$ of dimension $k d$ comprising $k$ disjoint copies of $\mathcal{V}^{\prime}$ (each copy works on its own counters); let $(q, j)$ denote the control state $q$ of $\mathcal{V}^{\prime}$ in the $j$-th copy $(j \in[1, k])$.

Finally, we let $\mathcal{V}^{\prime \prime \prime}$ arise from $\mathcal{V}^{\prime \prime}$ by adding transitions $\left(q_{j}, j\right) \stackrel{0}{\rightarrow}\left(q_{0}, j+1\right)$, for $j \in[1, k-1]$. We observe that

$$
q_{0}(\mathbf{0}) \stackrel{*}{\rightarrow}_{\mathcal{V}^{\prime}} q_{j}\left(v_{j}\right) \text { for all } j \in[1, k] \text { iff }\left(q_{0}, 1\right)(\mathbf{0}) \stackrel{*}{\rightarrow}_{\mathcal{V}^{\prime \prime \prime}}\left(q_{k}, k\right)\left(v_{1}, \ldots, v_{k}\right) .
$$

We have thus shown the claimed logspace reduction.

We leave open the question if the FMIC co-finiteness problem can be similarly reduced to the reachability problem. Another open question is if reachability can be reduced to FMIC co-finiteness or FMIC co-emptiness. In the next section, we characterize the complexity of both problems for the case of single initial configurations. 


\section{Single Initial Configurations}

In this section we restrict our attention to the $S I C$ co-emptiness problem and the $S I C$ co-finiteness problem where SIC refers to "Single Initial Configuration"; the problem instances are thus restricted so that the given sets $C$ are singletons $\left(C=\left\{q_{0}\left(v_{0}\right)\right\}\right)$. In the rest of this section we prove the following theorem.

Theorem 6. Both the SIC co-finiteness problem and the SIC co-emptiness problem are EXPSPACE-complete.

We recall that the integers in the problem instances are presented in binary. Nevertheless the lower bound will be shown already for unary VASs (hence with no control states and with a unary presentation of integers).

We first recall two well-known EXPSPACE-complete problems for VASSs where the lower bound also holds for unary VASs.

Coverability. The coverability problem:

Instance: a $\operatorname{VASS} \mathcal{V}=(d, Q, \mathcal{A}, T), q_{0}, q_{1} \in Q, v_{0}, v_{1} \in \mathbb{N}^{d}$;

Question: is $q_{0}\left(v_{0}\right) \stackrel{*}{\rightarrow} q_{1}\left(\bar{v}_{1}\right)$ for some $\bar{v}_{1} \geq v_{1}$ ?

Boundedness. The boundedness problem:

Instance: a VASS $\mathcal{V}=(d, Q, \mathcal{A}, T), q_{0} \in Q, v_{0} \in \mathbb{N}^{d}$

Question: is $\left[q_{0}\left(v_{0}\right)\right\rangle$ finite?

The EXPSPACE-hardness results follow from [11] (see also, e.g. [3]), the upper bounds follow from [13]. A generalization of [13], extending a class of problems known to be in EXPSPACE, was given in [14], which was later corrected in [1].

\subsection{EXPSPACE-hardness}

Showing the hardness part of Theorem 6 is relatively straightforward; we reduce coverability in unary VASs (which we recalled as an EXPSPACE-complete problem) to both SIC co-finiteness and SIC co-emptiness by the following lemma.

Lemma 7. Given a unary $\operatorname{VAS} \mathcal{U}=(d, \mathcal{A})$ and $v_{0}, v_{1} \in \mathbb{N}^{d}$, there is a logspace construction yielding a unary $V A S \mathcal{U}^{\prime}=\left(d+1, \mathcal{A}^{\prime}\right)$ and $v_{0}^{\prime} \in \mathbb{N}^{d+1}$ such that:

a) if $v_{0} \stackrel{*}{\rightarrow} \mathcal{U} \bar{v}_{1}$ for some $\bar{v}_{1} \geq v_{1}$, then $\left[v_{0}^{\prime}\right\rangle_{\mathcal{U}^{\prime}}=\mathbb{N}^{d+1}$;

b) otherwise (when $v_{0} \stackrel{*}{\rightarrow} \mathcal{U} w$ implies $w \nsupseteq v_{1}$ ) the set $\mathbb{N}^{d+1} \backslash\left[v_{0}^{\prime}\right\rangle_{\mathcal{U}^{\prime}}$ is infinite.

Proof. Let us assume a unary $\operatorname{VAS} \mathcal{U}=(d, \mathcal{A})$ and vectors $v_{0}, v_{1} \in \mathbb{N}^{d}$. We consider $\mathcal{U}^{\prime}=\left(d+1, \mathcal{A}^{\prime}\right)$ and $v_{0}^{\prime}=\left(v_{0}, 0\right)$ where

$$
\mathcal{A}^{\prime}=\{(\mathbf{a}, 0) \mid \mathbf{a} \in \mathcal{A}\} \cup\left\{\mathbf{b}_{1}, \mathbf{b}_{2}\right\} \cup\left\{\mathbf{c}_{j} \mid j \in[1, d]\right\} \cup\left\{-\mathbf{e}_{j} \mid j \in[1, d]\right\}
$$




$$
\text { for } \mathbf{b}_{1}=\left(-v_{1}, 2\right), \mathbf{b}_{2}=\left(v_{0},-1\right), \mathbf{c}_{j}=\mathbf{e}_{j}-\mathbf{e}_{d+1} \text {. }
$$

It suffices to verify that the points a) and b) are satisfied (for $\mathcal{U}^{\prime}$ and $v_{0}^{\prime}$ ):

a) Suppose $v_{0} \stackrel{\alpha}{\rightarrow} \mathcal{U} \bar{v}_{1}$ for some $\bar{v}_{1} \geq v_{1}$ and $\alpha=\mathbf{a}_{1} \mathbf{a}_{2} \cdots \mathbf{a}_{m}$.

For $\alpha^{\prime}=\left(\mathbf{a}_{1}, 0\right)\left(\mathbf{a}_{2}, 0\right) \cdots\left(\mathbf{a}_{m}, 0\right)$, in $\mathcal{U}^{\prime}$ we then have

$$
\left(v_{0}, 0\right) \stackrel{\alpha^{\prime}}{\longrightarrow}\left(\bar{v}_{1}, 0\right) \stackrel{\mathbf{b}_{1}}{\longrightarrow}\left(\bar{v}_{1}-v_{1}, 2\right) \stackrel{\mathbf{b}_{2}}{\longrightarrow}\left(v_{0}+\bar{v}_{1}-v_{1}, 1\right) .
$$

By monotonicity, for any $k \in \mathbb{N}$ we have

$$
v_{0}^{\prime}=\left(v_{0}, 0\right) \stackrel{\left(\alpha^{\prime} \mathbf{b}_{1} \mathbf{b}_{2}\right)^{k}}{\longrightarrow} w_{k}=\left(v_{0}+k\left(\bar{v}_{1}-v_{1}\right), k\right)
$$

hence $w_{k}(d+1)=k$. For any $w \in \mathbb{N}^{d+1}$ and the sum $k=\sum_{j \in[1, d+1]} w(j)$ we have $w_{k} \stackrel{*}{\rightarrow} w$; indeed, in $w_{k}$ we can first empty (i.e., set to zero) all components $j \in[1, d]$ by using actions $-\mathbf{e}_{j}(j \in[1, d])$, and then distribute the $k$ tokens from component $d+1$ by the actions $\mathbf{c}_{j}$ so that $w$ is reached. Hence $\left[v_{0}^{\prime}\right\rangle_{\mathcal{U}^{\prime}}=\mathbb{N}^{d+1}$.

b) Suppose there is no $\bar{v}_{1} \geq v_{1}$ such that $v_{0} \stackrel{*}{\rightarrow}_{\mathcal{U}} \bar{v}_{1}$. Then for any $w \in\left[v_{0}^{\prime}\right\rangle_{\mathcal{U}^{\prime}}$ we have $w \nsupseteq\left(v_{1}, 0\right)$ and $w(d+1)=0$, since the actions $\mathbf{b}_{1}, \mathbf{b}_{2}, \mathbf{c}_{j}$ are dead (they cannot get enabled from $\left.v_{0}^{\prime}\right)$; indeed, by monotonicity the actions $-\mathbf{e}_{j}$ cannot help to cover $\left(v_{1}, 0\right)$ from $v_{0}^{\prime}$. Hence the set $\mathbb{N}^{d+1} \backslash\left[v_{0}^{\prime}\right\rangle_{\mathcal{U}^{\prime}}$ is infinite.

\subsection{EXPSPACE-membership.}

We now prove the EXPSPACE-membership claimed by Theorem 6 . This is more involved; besides a closer look at the results in [13], we will also use the following result from [9], from which we derive Lemma 9.

Theorem 8 ([9]). Given a $V A S S \mathcal{V}=(d, Q, \mathcal{A}, T)$ and two configurations $q_{0}\left(v_{0}\right)$ and $q_{1}\left(v_{1}\right)$ reachable one from the other $\left(i . e ., q_{0}\left(v_{0}\right) \stackrel{*}{\rightarrow} q_{1}\left(v_{1}\right) \stackrel{*}{\rightarrow} q_{0}\left(v_{0}\right)\right)$, there is a word $\alpha \in \mathcal{A}^{*}$ such that

a) $q_{0}\left(v_{0}\right) \stackrel{\alpha}{\rightarrow} q_{1}\left(v_{1}\right)$, and

b) $|\alpha| \leq 6 \cdot(d+3)^{2} \cdot x^{45(d+3)^{d+5}}$ where $x=1+2|Q|+2\|\mathcal{A}\|+2\left\|v_{0}\right\|+\left\|v_{1}\right\|$.

Proof. Theorem 10.1 of [9] states that for every pair $\left(v_{0}^{\prime}, v_{1}^{\prime}\right)$ of configurations of a $\operatorname{VAS}\left(p, \mathcal{A}^{\prime}\right)$ that are reachable one from the other there is a word $\alpha^{\prime} \in\left(\mathcal{A}^{\prime}\right)^{*}$ such that:

$$
v_{0}^{\prime} \stackrel{\alpha^{\prime}}{\rightarrow} v_{1}^{\prime} \quad \text { and } \quad\left|\alpha^{\prime}\right| \leq 17 p^{2} y^{15 p^{p+2}}
$$

where $y=\left(1+2\left\|\mathcal{A}^{\prime}\right\|\right)\left(1+\left\|v_{0}^{\prime}\right\|+\left\|v_{1}^{\prime}-v_{0}^{\prime}\right\|\right)$. We extend this result to a VASS $(d, Q, \mathcal{A}, T)$ by encoding it as a $\operatorname{VAS}\left(p, \mathcal{A}^{\prime}\right)$ using [6, Lemma 2.1]. With this encoding, $p=d+3,\left\|\mathcal{A}^{\prime}\right\| \leq \max \{\|\mathcal{A}\|,|Q| \cdot(|Q|-1)\}$ and the encodings of $q_{0}\left(v_{0}\right)$ and $q_{1}\left(v_{1}\right)$ provide vectors $v_{0}^{\prime}, v_{1}^{\prime}$ satisfying $\left\|v_{0}^{\prime}\right\| \leq\left\|v_{0}\right\|+|Q|$ and $\left\|v_{1}^{\prime}-v_{0}^{\prime}\right\|=$ $\left\|v_{1}-v_{0}\right\| \leq\left\|v_{1}\right\|+\left\|v_{0}\right\|$. It follows that $\left(1+2\left\|\mathcal{A}^{\prime}\right\|\right) \leq x^{2}$ and $\left(1+\left\|v_{0}^{\prime}\right\|+\| v_{1}^{\prime}-\right.$ $\left.v_{0}^{\prime} \|\right) \leq x$. Thus $y$ is bounded by $x^{3}$. Finally, since the effect of an action of the VASS is simulated by three actions of the simulating VAS, we deduce that there exists a word $\alpha \in \mathcal{A}^{*}$ such that $q_{0}\left(v_{0}\right) \stackrel{\alpha}{\rightarrow} q_{1}\left(v_{1}\right)$ and such that $|\alpha| \leq \frac{1}{3}\left|\alpha^{\prime}\right|$. We derive the bound on $|\alpha|$ by observing that $\frac{17}{3} \leq 6$. 
Pumpability of components. Given a VASS $\mathcal{V}=(d, Q, \mathcal{A}, T)$, we say that component $i \in[1, d]$ is pumpable in $q(v)$ if $q(v) \stackrel{*}{\rightarrow} q\left(v+k \mathbf{e}_{i}\right)$ for some $k \geq 1$.

Lemma 9. For any $\operatorname{VASS} \mathcal{V}=(d, Q, \mathcal{A}, T)$ and any $q \in Q, v \in \mathbb{N}^{d}, i \in[1, d]$ where component $i$ is pumpable in $q(v)$ there is $\alpha \in \mathcal{A}^{*}$ such that

a) $q(v) \stackrel{\alpha}{\rightarrow} q\left(v+k \mathbf{e}_{i}\right)$ for some $k \geq 1$, and

b) $|\alpha| \leq 6 \cdot(d+3)^{2} \cdot x^{45(d+3)^{d+5}}$ where $x=2+2|Q|+2\|\mathcal{A}\|+3\|v\|$.

The trivial fact $k \leq|\alpha| \cdot\|\mathcal{A}\|$ thus also yields a double-exponential bound on $k$.

Proof. We consider a VASS $\mathcal{V}=(d, Q, \mathcal{A}, T)$ and assume $q(v) \stackrel{*}{\rightarrow} \mathcal{V} q\left(v+k \mathbf{e}_{i}\right)$ where $k \geq 1$. For the VASS $\mathcal{V}^{\prime}$ arising from $\mathcal{V}$ by adding (action $-\mathbf{e}_{i}$ and) the transition $q \stackrel{-\mathbf{e}_{i}}{\longrightarrow} q$ we get

$$
q(v) \stackrel{*}{\rightarrow} q\left(v+k \mathbf{e}_{i}\right) \stackrel{-\mathbf{e}_{i}}{\longrightarrow} \cdots \stackrel{-\mathbf{e}_{i}}{\longrightarrow} q\left(v+\mathbf{e}_{i}\right) \stackrel{-\mathbf{e}_{i}}{\longrightarrow} q(v) ;
$$

hence $q(v)$ and $q\left(v+\mathbf{e}_{i}\right)$ are reachable one from the other (they are in the reversible-reachability relation) in $\mathcal{V}^{\prime}$. Using Theorem 8, we derive that

$$
q(v) \stackrel{\alpha}{\rightarrow} \mathcal{V}^{\prime} q\left(v+\mathbf{e}_{i}\right)
$$

for some $\alpha \in\left(\mathcal{A} \cup\left\{-\mathbf{e}_{i}\right\}\right)^{*}$ that is bounded as in the point b) of the claim.

If $\alpha$ in (2) is $\mathbf{a}_{1} \mathbf{a}_{2} \cdots \mathbf{a}_{m}$, then there are states $q_{1}, q_{2}, \ldots, q_{m-1}$ such that

$$
q(v) \stackrel{\mathbf{a}_{1}}{\longrightarrow} q_{1}\left(v_{1}\right) \stackrel{\mathbf{a}_{2}}{\longrightarrow} q_{2}\left(v_{2}\right) \stackrel{\mathbf{a}_{3}}{\longrightarrow} \cdots q_{m-1}\left(v_{m-1}\right) \stackrel{\mathbf{a}_{m}}{\longrightarrow} q\left(v+\mathbf{e}_{i}\right)
$$

for the corresponding $v_{j}(j \in[1, m-1])$. We can view (3) as a sequence of transitions; let $\ell \geq 0$ be the number of occurrences of the transition $q \stackrel{-\mathbf{e}_{i}}{\longrightarrow} q$ in (3). Due to monotonicity, we can omit these occurrences and keep performability: we get

$$
q(v) \stackrel{\mathbf{a}_{i_{1}} \mathbf{a}_{i_{2}} \cdots \mathbf{a}_{i_{m-\ell}}}{\longrightarrow} \mathcal{V} q\left(v+(\ell+1) \mathbf{e}_{i}\right)
$$

for the sequence $\mathbf{a}_{i_{1}} \mathbf{a}_{i_{2}} \cdots \mathbf{a}_{i_{m-\ell}}$ arising from $\mathbf{a}_{1} \mathbf{a}_{2} \cdots \mathbf{a}_{m}$ by omitting the respective $\ell$ occurrences of $-\mathbf{e}_{i}$. The proof is thus finished.

We derive the following important corollary:

Corollary 10. There is an exponential-space algorithm that, given a VASS $\mathcal{V}=$ $(d, Q, \mathcal{A}, T)$ and $q(v)$, decides if all components $i \in[1, d]$ are pumpable in $q(v)$, and in the positive case provides an (at most double-exponential) number $n \geq 1$ such that $q(v) \stackrel{*}{\rightarrow} q\left(v+n \mathbf{e}_{i}\right)$ for each $i \in[1, d]$.

Proof. It suffices to consider a nondeterministic algorithm trying to find, for each $i \in[1, d]$ separately, $\alpha_{i}$ with length bounded as in Lemma 9 such that $q(v) \stackrel{\alpha_{i}}{\longrightarrow} q\left(v+k_{i} \mathbf{e}_{i}\right)$ for some $k_{i} \geq 1$. The algorithm just traverses along (a guessed bounded) $\alpha_{i}$, keeping only the current configuration in memory; hence exponential space is sufficient.

By monotonicity, $q(v) \stackrel{*}{\rightarrow} q\left(v+k_{i} \mathbf{e}_{i}\right)$ implies that $q(v) \stackrel{*}{\rightarrow} q\left(v+x k_{i} \mathbf{e}_{i}\right)$ for all $x \geq 1$. Hence if $k_{i} \geq 1$ for all $i \in[1, d]$ are found, then the least common multiple (or even simply the product) of all $k_{i}, i \in[1, d]$, can be taken as the claimed number $n$. 
Before giving the algorithm deciding SIC co-emptiness we introduce some useful natural notions, namely a notion of "reversing a VASS" (letting its computations run backwards), and a notion of "transforming a VASS modulo $n$ " (where the component-values are divided by $n$ while the remainders are kept in the control states).

Reversed VASS. To a VASS $\mathcal{V}=(d, Q, \mathcal{A}, T)$ we associate its reversed VASS

$$
\mathcal{V}^{\leftarrow}=\left(d, Q,-\mathcal{A}, T^{\leftarrow}\right)
$$

where $-\mathcal{A}=\{-\mathbf{a} \mid \mathbf{a} \in \mathcal{A}\}$ and $T^{\leftarrow}=\left\{q^{\prime} \stackrel{-\mathbf{a}}{\rightarrow} q \mid q \stackrel{\mathbf{a}}{\rightarrow} q^{\prime}\right.$ is in $\left.T\right\}$.

The next proposition can be easily verified by induction on $m$.

Proposition 11. For any VASS $\mathcal{V}$ and $m \geq 1$, we have

$$
\begin{aligned}
& q_{0}\left(v_{0}\right) \stackrel{\mathbf{a}_{1}}{\longrightarrow} q_{1}\left(v_{1}\right) \stackrel{\mathbf{a}_{2}}{\longrightarrow} q_{2}\left(v_{2}\right) \stackrel{\mathbf{a}_{3}}{\longrightarrow} \cdots q_{m-1}\left(v_{m-1}\right) \stackrel{\mathbf{a}_{m}}{\longrightarrow} q_{m}\left(v_{m}\right) \text { in } \mathcal{V} \\
& q_{m}\left(v_{m}\right) \stackrel{-\mathbf{a}_{m}}{\longrightarrow} q_{m-1}\left(v_{m-1}\right) \stackrel{-\mathbf{a}_{m-1}}{\longrightarrow} \cdots q_{1}\left(v_{1}\right) \stackrel{-\mathbf{a}_{1}}{\longrightarrow} q_{0}\left(v_{0}\right) \text { in } \mathcal{V}^{\leftarrow} .
\end{aligned}
$$

Modulo- $\boldsymbol{n}$ VASS. Given a VASS $\mathcal{V}=(d, Q, \mathcal{A}, T)$ and $n \geq 1$, we put

$$
\mathcal{V}_{(n)}=\left(d, Q \times\{0,1, \ldots, n-1\}^{d}, \mathcal{A}^{\prime}, T_{(n)}\right)
$$

where $T_{(n)}$ arises as follows:

each transition $q \stackrel{\mathbf{a}}{\rightarrow} q^{\prime}$ in $T$ and each $u \in\{0,1, \ldots, n-1\}^{d}$ determines

$$
\text { the transition }(q, u) \stackrel{\mathbf{a}^{\prime}}{\rightarrow}\left(q^{\prime}, u^{\prime}\right) \text { in } T_{(n)}
$$

where $u^{\prime}$ and $\mathbf{a}^{\prime}$ are the unique vectors such that $u+\mathbf{a}=u^{\prime}+n \mathbf{a}^{\prime}$ and $u^{\prime} \in$ $\{0,1, \ldots, n-1\}^{d}$. The set $\mathcal{A}^{\prime}$ is simply $\left\{\mathbf{a}^{\prime} \mid\left((q, u) \stackrel{\mathbf{a}^{\prime}}{\rightarrow}\left(q^{\prime}, u^{\prime}\right)\right) \in T_{(n)}\right\}$.

The next proposition is again easily verifiable by induction on $m$.

Proposition 12. For any VASS $\mathcal{V}, n \geq 1$, and $m \geq 1$, we have

$$
\begin{gathered}
q_{0}\left(v_{0}\right) \stackrel{\mathbf{a}_{1}}{\longrightarrow} q_{1}\left(v_{1}\right) \stackrel{\mathbf{a}_{2}}{\longrightarrow} q_{2}\left(v_{2}\right) \stackrel{\mathbf{a}_{3}}{\longrightarrow} \cdots q_{m-1}\left(v_{m-1}\right) \stackrel{\mathbf{a}_{m}}{\longrightarrow} q_{m}\left(v_{m}\right) \text { in } \mathcal{V} \\
\left(q_{0}, u_{0}\right)\left(v_{0}^{\prime}\right) \stackrel{\mathbf{a}_{1}^{\prime}}{\longrightarrow}\left(q_{1}, u_{1}\right)\left(v_{1}^{\prime}\right) \stackrel{\mathbf{a}_{2}^{\prime}}{\longrightarrow} \cdots \stackrel{\mathbf{a}_{m}^{\prime}}{\longrightarrow}\left(q_{m}, u_{m}\right)\left(v_{m}^{\prime}\right) \text { in } \mathcal{V}_{(n)}
\end{gathered}
$$

where $u_{j}+n v_{j}^{\prime}=v_{j}$ for every $j \in[0, m]$ (and $u_{j-1}+\mathbf{a}_{j}=u_{j}+n \mathbf{a}_{j}^{\prime}$ for every $j \in[1, m])$.

Algorithm deciding SIC co-emptiness. We define the following algorithm.

\section{Algorithm ALG-Co-EmPT}

Input: a VASS $\mathcal{V}=(d, Q, \mathcal{A}, T)$ and a configuration $q_{0}\left(v_{0}\right)$.

Output: YES if $\left[q_{0}\left(v_{0}\right)\right\rangle=Q \times \mathbb{N}^{d}$, and NO otherwise. 
1. Check if each component $i \in[1, d]$ is pumpable in $q_{0}\left(v_{0}\right)$, and in the positive case compute an (at most double-exponential) number $n$ as described in Corollary 10 (hence $q_{0}\left(v_{0}\right) \stackrel{*}{\rightarrow} q_{0}\left(v_{0}+n \mathbf{e}_{i}\right)$ for each $\left.i \in[1, d]\right)$.

In the negative case (when some component is not pumpable) return NO.

2. Let $\mathcal{V}^{\prime}$ be the VASS $\mathcal{V}^{\prime}=\left(\mathcal{V}^{\leftarrow}\right)_{(n)}=\left(d, Q \times\{0,1, \ldots, n-1\}^{d}, \mathcal{A}^{\prime}, T^{\prime}\right)$ i.e., the reversed VASS modulo $n$, where $n$ is computed in the point 1$\}$.

Create the configuration $\left(q_{0}, u_{0}\right)\left(v_{0}^{\prime}\right)$ of $\mathcal{V}^{\prime}$ corresponding to the configuration $q_{0}\left(v_{0}\right)$ of $\mathcal{V}$ (hence $v_{0}=u_{0}+n v_{0}^{\prime}$ ).

3. For each control state $(q, u)$ of $\mathcal{V}^{\prime}$ check if $(q, u)(\mathbf{0})$ covers $\left(q_{0}, u_{0}\right)\left(v_{0}^{\prime}\right)$ (in $\left.\mathcal{V}^{\prime}\right)$, i.e., if $(q, u)(\mathbf{0}) \stackrel{*}{\rightarrow} \mathcal{V}^{\prime}\left(q_{0}, u_{0}\right)(\bar{v})$ for some $\bar{v} \geq v_{0}^{\prime}$.

If the answer is negative for some $(q, u)$, then return $\mathrm{NO}$, otherwise (when all $(q, u)(\mathbf{0})$ cover $\left.\left(q_{0}, u_{0}\right)\left(v_{0}^{\prime}\right)\right)$ return YES.

\section{Correctness and exponential-space complexity of Alg-Co-Empt.}

Lemma 13. Algorithm ALG-Co-EMPT satisfies its specification (i.e., returns $Y E S$ if $\left[q_{0}\left(v_{0}\right)\right\rangle=Q \times \mathbb{N}^{d}$, and $N O$ otherwise).

Proof. If Alg-Co-Empt, when given $\mathcal{V}=(d, Q, \mathcal{A}, T)$ and $q_{0}\left(v_{0}\right)$, returns NO in the point 1 , then for some $i \in[1, d]$ we have $q_{0}\left(v_{0}\right) \stackrel{\text { 光 }}{\rightarrow} q_{0}\left(v_{0}+x \mathbf{e}_{i}\right)$ for all $x \geq 1$; therefore the set $\left(Q \times \mathbb{N}^{d}\right) \backslash\left[q_{0}\left(v_{0}\right)\right\rangle_{\mathcal{V}}$ is nonempty and even infinite.

Suppose now that the test in the point 1 has been positive, and a respective number $n$ has been computed.

Assume first that $\left[q_{0}\left(v_{0}\right)\right\rangle_{\mathcal{V}}=Q \times \mathbb{N}^{d}$ and let us show that the algorithm returns YES. Let $(q, u)$ be a control state of $\mathcal{V}^{\prime}=\left(\mathcal{V}^{\leftarrow}\right)_{(n)}$. Since $\left[q_{0}\left(v_{0}\right)\right\rangle_{\mathcal{V}}=$ $Q \times \mathbb{N}^{d}$, we have $q_{0}\left(v_{0}\right) \stackrel{*}{\rightarrow} \mathcal{V} q(u)$. It follows that $(q, u)(\mathbf{0}) \stackrel{*}{\rightarrow} \mathcal{V}^{\prime}\left(q_{0}, u_{0}\right)\left(v_{0}^{\prime}\right)$, which also entails that $(q, u)(\mathbf{0})$ covers $\left(q_{0}, u_{0}\right)\left(v_{0}^{\prime}\right)$ in $\mathcal{V}^{\prime}$. We have proved that the algorithm returns YES.

Conversely, we assume that the algorithm returns YES and we prove that $\left[q_{0}\left(v_{0}\right)\right\rangle_{\mathcal{V}}=Q \times \mathbb{N}^{d}$. Let $q(v)$ be a configuration of $\mathcal{V}$ and let $(q, u)\left(v^{\prime}\right)$ be the corresponding configuration in $\mathcal{V}^{\prime}$, i.e., $v=u+n v^{\prime}$. Since $(q, u)(\mathbf{0})$ covers $\left(q_{0}, u_{0}\right)\left(v_{0}^{\prime}\right)$, there exists $\bar{v}_{0}^{\prime} \geq v_{0}^{\prime}$ such that $(q, u)(\mathbf{0}) \stackrel{*}{\rightarrow} \mathcal{V}^{\prime}\left(q_{0}, u_{0}\right)\left(\bar{v}_{0}^{\prime}\right)$. It follows that $q_{0}\left(u_{0}+n \bar{v}_{0}^{\prime}\right) \stackrel{*}{\rightarrow} \mathcal{V} q(u)$. By monotonicity, we derive that

$$
q_{0}\left(u_{0}+n \bar{v}_{0}^{\prime}+n v^{\prime}\right) \stackrel{*}{\rightarrow} \mathcal{V} q\left(u+n v^{\prime}\right)=q(v) .
$$

By the definition of $n$, we get

$$
q_{0}\left(v_{0}\right) \stackrel{*}{\rightarrow} \mathcal{V} q_{0}\left(v_{0}+n\left(\bar{v}_{0}^{\prime}-v_{0}^{\prime}\right)+n v^{\prime}\right)=q_{0}\left(u_{0}+n \bar{v}_{0}^{\prime}+n v^{\prime}\right) .
$$

We have proved that $q_{0}\left(v_{0}\right) \stackrel{*}{\rightarrow} \mathcal{V} q(v)$, and thus $\left[q_{0}\left(v_{0}\right)\right\rangle_{\mathcal{V}}=Q \times \mathbb{N}^{d}$.

We still need to show that ALG-Co-EMPT works in exponential space (Lemma 15). We first give a straightforward extension to VASSs of a result formulated in [13] for VASs. 
Proposition 14. For any $V A S S \mathcal{V}=(d, Q, \mathcal{A}, T)$ and any configurations $q_{0}\left(v_{0}\right)$ and $q_{1}\left(v_{1}\right)$, if $q_{0}\left(v_{0}\right) \stackrel{*}{\rightarrow} q_{1}\left(v_{1}\right)$ then $q_{0}\left(v_{0}\right) \stackrel{\alpha}{\rightarrow} q_{1}\left(\bar{v}_{1}\right)$ for some $\bar{v}_{1} \geq v_{1}$ and $\alpha \in \mathcal{A}^{*}$ such that $|\alpha|<x^{(d+1) !}$, where $x=|Q| \cdot\left(1+\|\mathcal{A}\|+\left\|v_{1}\right\|\right)$.

Proof. The bounds given in [13] for VASs are easily extended to VASSs. Instead of giving a full proof, we only explain how to adapt the proof of [13] to deal with control states.

The notions of paths, of $i$-bounded sequences and of $i$-covering sequences from [13, pages 224-225] are extended with control states in the obvious way. For each $q \in Q$ and $v \in \mathbb{Z}^{d}$, define $m(i, q, v)$ to be the length of the shortest $i$-bounded, $i$-covering path in $\mathcal{V}$ starting from $q(v)$, with the convention that $m(i, q, v)=0$ if there is none.

Now define $f(i)=\max \left\{m(i, q, v) \mid q \in Q, v \in \mathbb{Z}^{d}\right\}$. With the same reasoning as in [13, Lemma 3.4], we get that

$$
f(0) \leq|Q| \text { and } f(i+1) \leq|Q| \cdot\left(\max \left\{\|\mathcal{A}\|,\left\|v_{1}\right\|\right\} \cdot f(i)\right)^{i+1}+f(i) .
$$

It follows that $f(i+1) \leq(x f(i))^{i+1}$. An immediate induction on $i$ yields that $f(i) \leq x^{(i+1) !}$. In particular, we get that $m\left(d, q_{0}, v_{0}\right) \leq f(d) \leq x^{(d+1) !}$. Now, if $q_{0}\left(v_{0}\right) \stackrel{*}{\rightarrow} q_{1}\left(v_{1}\right)$ then $0<m\left(d, q_{0}, v_{0}\right)$. This entails that $q_{0}\left(v_{0}\right) \stackrel{\alpha}{\rightarrow} q_{1}\left(\bar{v}_{1}\right)$ for some $\bar{v}_{1} \geq v_{1}$ and $\alpha \in \mathcal{A}^{*}$ such that $|\alpha|=m\left(d, q_{0}, v_{0}\right)-1<x^{(d+1) !}$.

Lemma 15. Algorithm ALG-Co-EMPT works (i.e., can be implemented to work) in exponential space.

Proof. The point 1 of ALG-Co-EMPT, including the binary presentation of the computed number $n$, can be performed in exponential space, w.r.t. the size of the binary presentation of the input $\mathcal{V}=(d, Q, \mathcal{A}, T)$ and $q_{0}\left(v_{0}\right)$; this follows by Corollary 10.

The VASS $\mathcal{V}^{\prime}=\left(\mathcal{V}^{\leftarrow}\right)_{(n)}$ in the point 2 is not needed to be constructed explicitly. The algorithm creates the configuration $\left(q_{0}, u_{0}\right)\left(v_{0}^{\prime}\right)$ and then stepwise generates the control states $(q, u)\left(q \in Q, u \in\{0,1, \ldots, n-1\}^{d}\right)$ of $\mathcal{V}^{\prime}$ and checks if $(q, u)(\mathbf{0})$ covers $\left(q_{0}, u_{0}\right)\left(v_{0}^{\prime}\right)$ in $\mathcal{V}^{\prime}$.

It thus suffices to show that checking if $(q, u)(\mathbf{0})$ covers $\left(q_{0}, u_{0}\right)\left(v_{0}^{\prime}\right)$ (i.e., if $(q, u)(\mathbf{0}) \stackrel{*}{\rightarrow} \mathcal{V}^{\prime}\left(q_{0}, u_{0}\right)(\bar{v})$ for some $\left.\bar{v} \geq v_{0}^{\prime}\right)$ can be done in exponential space (w.r.t. the binary presentation of $\mathcal{V}=(d, Q, \mathcal{A}, T)$ and $\left.q_{0}\left(v_{0}\right)\right)$. By Prop. 14, it is enough to search for witnesses of coverability $(q, u)(\mathbf{0}) \stackrel{\alpha}{\rightarrow}\left(q_{0}, u_{0}\right)(\bar{v})$ of length $|\alpha|<x^{(d+1) !}$, where $x=|Q| n^{d} \cdot\left(1+\left\|\mathcal{A}^{\prime}\right\|+\left\|v_{0}^{\prime}\right\|\right)$. Since $n$ is at most double-exponential, $x^{(d+1) !}$ is also at most double-exponential. As in the proof of Corollary 10, the algorithm just traverses along (a guessed bounded) $\alpha$, keeping only the current configuration in memory; so exponential space is sufficient.

Algorithm deciding SIC co-finiteness. We will adjust the algorithm ALGCo-EMPT so that, given $\mathcal{V}=(d, Q, \mathcal{A}, T)$ and $q_{0}\left(v_{0}\right)$, it answers YES iff the set $\left(Q \times \mathbb{N}^{d}\right) \backslash\left[q_{0}\left(v_{0}\right)\right\rangle$ is finite; this can happen even if some $(q, u)(\mathbf{0})$ does not cover $\left(q_{0}, u_{0}\right)\left(v_{0}^{\prime}\right)$ in $\mathcal{V}^{\prime}$. Informally speaking, it suffices to check if $(q, u)(\mathbf{0})$ covers 
$\left(q_{0}, u_{0}\right)\left(v_{0}^{\prime}\right)$ whenever we "ignore" one-component of $\mathbf{0}$, making it "arbitrarily large".

By $\omega$ we denote an "infinite amount", satisfying $z<\omega$ and $z+\omega=\omega+z=\omega$ for all $z \in \mathbb{Z}$. Given $\mathcal{V}=(d, Q, \mathcal{A}, T)$, by the set of extended configurations we mean the set $Q \times(\mathbb{N} \cup\{\omega\})^{d}$; the relations $q(v) \stackrel{\text { a }}{\rightarrow} q^{\prime}\left(v^{\prime}\right), q(v) \stackrel{\alpha}{\rightarrow} q^{\prime}\left(v^{\prime}\right)\left(\alpha \in \mathcal{A}^{*}\right)$, and $q(v) \stackrel{*}{\rightarrow} q^{\prime}\left(v^{\prime}\right)$ are then naturally extended to the relations on $Q \times(\mathbb{N} \cup\{\omega\})^{d}$. (Hence, e.g., if $q(v) \stackrel{\alpha}{\rightarrow} q^{\prime}\left(v^{\prime}\right)$ then $v(i)=\omega$ iff $v^{\prime}(i)=\omega$, for any $i \in[1, d]$.)

Let us now consider the following algorithm.

Algorithm ALG-Co-FINIT

Input: a $\operatorname{VASS} \mathcal{V}=(d, Q, \mathcal{A}, T)$ and a configuration $q_{0}\left(v_{0}\right)$.

Output: YES if $\left(Q \times \mathbb{N}^{d}\right) \backslash\left[q_{0}\left(v_{0}\right)\right\rangle$ is finite, and NO otherwise.

1. As in Alg-Co-Empt.

2. As in Alg-Co-Empt.

3. For each control state $(q, u)$ of $\mathcal{V}^{\prime}$ and each $i \in[1, d]$ check if $(q, u)\left(\omega \mathbf{e}_{i}\right)$ covers $\left(q_{0}, u_{0}\right)\left(v_{0}^{\prime}\right)$ (in $\left.\mathcal{V}^{\prime}\right)$, i.e., if

$$
(q, u)\left(\omega \mathbf{e}_{i}\right) \stackrel{*}{\rightarrow} \mathcal{V}^{\prime}\left(q_{0}, u_{0}\right)(\bar{v}) \text { for some } \bar{v} \geq v_{0}^{\prime} ;
$$

by $\omega \mathbf{e}_{i}$ we denote the $d$-dimensional vector where the $i$-th component is $\omega$ and the other components are zero.

If the answer is negative for some $(q, u)$ and $i \in[1, d]$, then return NO, otherwise (when all $(q, u)\left(\omega \mathbf{e}_{i}\right)$ cover $\left.\left(q_{0}, u_{0}\right)\left(v_{0}^{\prime}\right)\right)$ return YES.

\section{Correctness and exponential-space complexity of Alg-Co-Finit.}

Lemma 16. Algorithm ALG-CO-FINIT satisfies its specification (i.e., returns $Y E S$ if $\left(Q \times \mathbb{N}^{d}\right) \backslash\left[q_{0}\left(v_{0}\right)\right\rangle$ is finite, and $N O$ otherwise).

Proof. We reason analogously as in the proof of Lemma 13. We have already noted that if NO is returned in the point 1 , then $\left(Q \times \mathbb{N}^{d}\right) \backslash\left[q_{0}\left(v_{0}\right)\right\rangle$ is infinite.

Assume first that $\left[q_{0}\left(v_{0}\right)\right\rangle_{\mathcal{V}}$ is co-finite and let us show that the algorithm returns YES. Let $(q, u)$ be a control state of $\mathcal{V}^{\prime}$ and let $i \in[1, d]$. Since $\left[q_{0}\left(v_{0}\right)\right\rangle_{\mathcal{V}}$ is co-finite, there is a number $x \geq 1$ such that $q_{0}\left(v_{0}\right) \stackrel{*}{\rightarrow} \mathcal{V} q\left(u+n x \mathbf{e}_{i}\right)$. It follows that $(q, u)\left(x \mathbf{e}_{i}\right) \stackrel{*}{\rightarrow} \mathcal{V}^{\prime}\left(q_{0}, u_{0}\right)\left(v_{0}^{\prime}\right)$, which also entails that $(q, u)\left(\omega \mathbf{e}_{i}\right)$ covers $\left(q_{0}, u_{0}\right)\left(v_{0}^{\prime}\right)$ in $\mathcal{V}^{\prime}$. We have proved that the algorithm returns YES.

Assume now that the algorithm returns YES and let us prove that $\left[q_{0}\left(v_{0}\right)\right\rangle_{\mathcal{V}}$ is co-finite. Since $(q, u)\left(\omega \mathbf{e}_{i}\right)$ covers $\left(q_{0}, u_{0}\right)\left(v_{0}^{\prime}\right)$ in $\mathcal{V}^{\prime}$ for every control state $(q, u)$ of $\mathcal{V}^{\prime}$ and for every $i \in[1, d]$, there is a (large enough) number $x$ such that $(q, u)\left(x \mathbf{e}_{i}\right)$ covers $\left(q_{0}, u_{0}\right)\left(v_{0}^{\prime}\right)$ for every control state $(q, u)$ and every $i \in[1, d]$. Below we prove that every configuration $q(v)$ of $\mathcal{V}$ such that $\|v\| \geq n x$ is reachable from $q_{0}\left(v_{0}\right)$; this will entail that $\left[q_{0}\left(v_{0}\right)\right\rangle_{\mathcal{V}}$ is co-finite (i.e., $\left(Q \times \mathbb{N}^{d}\right) \backslash\left[q_{0}\left(v_{0}\right)\right\rangle_{\mathcal{V}}$ is finite).

We thus fix an arbitrary $q(v)$ and $i \in[1, d]$ such that $v(i) \geq n x$. Let $(q, u)\left(v^{\prime}\right)$ be the configuration of $\mathcal{V}^{\prime}$ corresponding to $q(v)$; hence $v=u+n v^{\prime}$. Since $(q, u)\left(x \mathbf{e}_{i}\right)$ covers $\left(q_{0}, u_{0}\right)\left(v_{0}^{\prime}\right)$ in $\mathcal{V}^{\prime}$, there is $\bar{v}_{0}^{\prime} \geq v_{0}^{\prime}$ such that 


$$
(q, u)\left(x \mathbf{e}_{i}\right) \stackrel{*}{\rightarrow} \mathcal{V}^{\prime}\left(q_{0}, u_{0}\right)\left(\bar{v}_{0}^{\prime}\right) ; \text { this entails } q_{0}\left(u_{0}+n \bar{v}_{0}^{\prime}\right) \stackrel{*}{\rightarrow} \mathcal{V} q\left(u+n x \mathbf{e}_{i}\right) .
$$

Since $v(i) \geq n x$, we have $v^{\prime}-x \mathbf{e}_{i} \geq \mathbf{0}$. By monotonicity we derive

$$
q_{0}\left(u_{0}+n \bar{v}_{0}^{\prime}+n\left(v^{\prime}-x \mathbf{e}_{i}\right)\right) \stackrel{*}{\rightarrow} \mathcal{V} q\left(u+n x \mathbf{e}_{i}+n\left(v^{\prime}-x \mathbf{e}_{i}\right)\right)=q(v) .
$$

By the definition of $n$, we get

$$
q_{0}\left(v_{0}\right) \stackrel{*}{\rightarrow} \mathcal{V} q_{0}\left(v_{0}+n\left(\bar{v}_{0}^{\prime}-v_{0}^{\prime}\right)+n\left(v^{\prime}-x \mathbf{e}_{i}\right)\right)=q_{0}\left(u_{0}+n \bar{v}_{0}^{\prime}+n\left(v^{\prime}-x \mathbf{e}_{i}\right)\right) .
$$

Hence we indeed have $q_{0}\left(v_{0}\right) \stackrel{*}{\rightarrow} \mathcal{V} q(v)$.

Lemma 17. Algorithm ALG-Co-Finit works (i.e., can be implemented to work) in exponential space.

Proof. This is analogous to the proof of Lemma 15. We just note that deciding if $(q, u)\left(\omega \mathbf{e}_{i}\right)$ covers $\left(q_{0}, u_{0}\right)\left(v_{0}^{\prime}\right)$ is even easier than deciding if $(q, u)(\mathbf{0})$ covers $\left(q_{0}, u_{0}\right)\left(v_{0}^{\prime}\right)$, since the $i$-th component can be simply ignored.

\section{Applications of the Co-Emptiness Problem}

A motivation for the study in this paper has been the decidability proof for structural liveness in [7], which is based on a particular version of the co-emptiness problem. We now give more details (in the framework of VASSs, which is equivalent to the framework of Petri nets used in [7]), and some partial complexity results. The main aim is to attract a further research effort on this topic, since the complexity of various related problems has not been answered. In particular, we have no nontrivial complexity bounds for the structural liveness problem (besides its decidability).

Assuming a VASS $\mathcal{V}=(d, Q, \mathcal{A}, T)$, we are now particularly interested in the co-emptiness of $[D\rangle_{\mathcal{V}}$ for downward closed sets $D \subseteq Q \times \mathbb{N}^{d}$, which constitute a subclass of semilinear sets. We use the notation

$$
\downarrow C=\left\{q(v) \mid v \leq v^{\prime} \text { for some } q\left(v^{\prime}\right) \in C\right\}
$$

for the downward closure of a set $C \subseteq Q \times \mathbb{N}^{d}$ (of configurations of $\mathcal{V}$ ). We say that $C \subseteq Q \times \mathbb{N}^{d}$ is downward closed if $\downarrow C=C$.

We write just $\downarrow q(v)$ instead of $\downarrow\{q(v)\}$.

Downward closed sets are semilinear since each such set can be presented as

$$
\downarrow q_{1}\left(\bar{v}_{1}\right) \cup \downarrow q_{2}\left(\bar{v}_{2}\right) \cup \cdots \cup \downarrow q_{m}\left(\bar{v}_{m}\right)
$$

for some $m \in \mathbb{N}$ and $\bar{v}_{i} \in(\mathbb{N} \cup\{\omega\})^{d}(i \in[1, m])$, where we put

$$
\downarrow q(\bar{v})=\left\{q(v) \mid v \leq \bar{v}, v \in \mathbb{N}^{d}\right\} .
$$


(Recall that $k<\omega$ for each $k \in \mathbb{N}$.)

Later we use another natural presentation of downward closed sets: for each $q \in Q$ we provide a constraint in the form of a (finite) conjunction of disjunctions of atomic constraints of the form $v(i) \leq c$ where $i \in[1, d]$ and $c \in \mathbb{N}$ (then $q(v) \in Q \times \mathbb{N}^{d}$ is in the set iff $v$ satisfies the constraint associated with $q$ ).

The DCIS co-emptiness problem where "DCIS" stands for "Downward Closed Initial Sets of configurations" (i.e., given $\mathcal{V}=(d, Q, \mathcal{A}, T)$ and a downward closed set $D \subseteq Q \times \mathbb{N}^{d}$, is $[D\rangle_{\mathcal{V}}=Q \times \mathbb{N}^{d}$ ?) is decidable by Theorem 3 (and the fact that $D$ is semilinear). The complexity is open, even the reductions to/from the reachability problem are unclear. Now we explain the previously mentioned motivation for such studies.

Liveness of transitions and configurations. We recall some standard definitions and facts. Given a $\operatorname{VASS} \mathcal{V}=(d, Q, \mathcal{A}, T)$,

- a transition $t \in T$ is enabled in a configuration $q(v)$ if $t$ is of the form $t: q \stackrel{\mathbf{a}}{\rightarrow} q^{\prime}$ and $v+\mathbf{a} \geq \mathbf{0}$

- a transition $t$ is live in $q(v)$ if for every $\bar{q}(\bar{v}) \in[q(v)\rangle$ there is $q^{\prime}\left(v^{\prime}\right) \in[\bar{q}(\bar{v})\rangle$ such that $t$ is enabled in $q^{\prime}\left(v^{\prime}\right)$;

- a transition $t$ is dead in $q(v)$ if there is no $q^{\prime}\left(v^{\prime}\right) \in[q(v)\rangle$ such that $t$ is enabled in $q^{\prime}\left(v^{\prime}\right)$.

We note that $t$ is not live in $q(v)$ iff $t$ is dead in some $q^{\prime}\left(v^{\prime}\right) \in[q(v)\rangle$.

The next proposition (which also defines $\mathcal{D}_{t, \mathcal{V}}$ and $\mathcal{D}_{\mathcal{V}}$ ) is obvious, due to monotonicity.

Proposition 18. Given a $V A S S \mathcal{V}=(d, Q, \mathcal{A}, T)$, for each $t \in T$ the set

$$
\mathcal{D}_{t, \mathcal{V}}=\{q(v) \mid t \text { is dead in } q(v)\}
$$

is downward closed. Hence also the set

$$
\mathcal{D}_{\mathcal{V}}=\{q(v) \mid \text { some } t \in T \text { is dead in } q(v)\}=\bigcup_{t \in T} \mathcal{D}_{t, \mathcal{V}}
$$

is downward closed.

Given a VASS $\mathcal{V}=(d, Q, \mathcal{A}, T)$, a configuration $q(v)$ is live if each $t \in T$ is live in $q(v)$, i.e., if $q(v) \stackrel{*}{7} \mathcal{V} \mathcal{D} \mathcal{V}$. A VASS $\mathcal{V}$ is structurally live if it has a live configuration, hence if the set

$$
\mathcal{L}_{\mathcal{V}}=\{q(v) \mid q(v) \text { is a live configuration of } \mathcal{V}\}
$$

is nonempty. While the membership problem for $\left(\mathcal{D}_{t, \mathcal{V}}\right.$ or $) \mathcal{D}_{\mathcal{V}}$ is essentially a version of the (non)coverability problem, which also allows to construct a natural presentation of the (downward closed) sets $\mathcal{D}_{t, \mathcal{V}}$ and $\mathcal{D}_{\mathcal{V}}$, the membership problem for $\mathcal{L}_{\mathcal{V}}$ is close to the reachability problem as was already noted by Hack [5] long time ago. 
The set $\mathcal{L}_{\mathcal{V}}$ is indeed more involved than $\mathcal{D}_{\mathcal{V}}$; it is obviously not downward closed but it is not upward closed either (in general), and it can be even nonsemilinear; we can refer to [7] for a concrete example, as well as for the following idea of decidability.

The structural liveness can be decided as follows. We recall the reversed VASS $\mathcal{V} \leftarrow$, and note that $\mathcal{V}$ is not structurally live iff $\left[\mathcal{D}_{\mathcal{V}}\right\rangle_{\mathcal{V}}$ is co-empty:

Proposition 19. For any $\operatorname{VASS} \mathcal{V}=(d, Q, \mathcal{A}, T)$ we have

$$
\left[\mathcal{D}_{\mathcal{V}}\right\rangle_{\mathcal{V} \leftarrow}=\left(Q \times \mathbb{N}^{d}\right) \backslash \mathcal{L}_{\mathcal{V}}
$$

Hence $\mathcal{V}$ is not structurally live iff $\left[\mathcal{D}_{\mathcal{V}}\right\rangle_{\mathcal{V} \leftarrow}=Q \times \mathbb{N}^{d}$.

Proof. We recall that $q(v)$ is not live iff $[q(v)\rangle_{\mathcal{V}} \cap \mathcal{D}_{\mathcal{V}} \neq \emptyset$ (i.e., iff $q(v) \stackrel{*}{\rightarrow} \mathcal{V} q^{\prime}\left(v^{\prime}\right)$ where some $t \in T$ is dead in $\left.q^{\prime}\left(v^{\prime}\right)\right)$. Hence $q(v)$ is not live iff $q^{\prime}\left(v^{\prime}\right) \stackrel{*}{\rightarrow} \mathcal{V} \leftarrow q(v)$ for some $q^{\prime}\left(v^{\prime}\right) \in \mathcal{D}_{\mathcal{V}}$ (using Proposition 11).

Therefore $\left[\mathcal{D}_{\mathcal{V}}\right\rangle_{\mathcal{V}^{\leftarrow}}=\left(Q \times \mathbb{N}^{d}\right) \backslash \mathcal{L}_{\mathcal{V}}$.

Proposition 19 allows us to decide structural liveness of a given VASS $\mathcal{V}=(d, Q, \mathcal{A}, T)$ by a reduction to the co-emptiness problem, using the abovementioned constructability of $\mathcal{D}_{\mathcal{V}}$.

Structural deadlock-freedom and DCIS co-emptiness. We have shown that the complementary problem of the structural liveness problem (hence "non structural liveness") can be reduced to the DCIS co-emptiness problem (with downward closed sets of initial configurations). However, we have no reduction from the latter problem to the former.

We now show that a special form of structural liveness, namely structural deadlock-freedom, is closely related to the DCIS co-emptiness problem. We use the previously mentioned presentation of downward closed sets by conjunctions of disjunctions of atomic constraints of the form $v(i) \leq c$ (for each $q \in Q$ ).

Given a VASS $\mathcal{V}=(d, Q, \mathcal{A}, T)$, a configuration $q(v)$ is deadlock-free if every configuration in $[q(v)\rangle$ enables some transition. A VASS $\mathcal{V}$ is structurally deadlock-free if it has a deadlock-free configuration. The structural deadlockfreedom problem asks, given a VASS $\mathcal{V}$, if $\mathcal{V}$ is structurally deadlock-free.

In the rest of this section we prove the following theorem.

Theorem 20. The complementary problem of the structural deadlock-freedom problem is polynomially interreducible with the DCIS co-emptiness problem. This entails that the structural deadlock-freedom problem is decidable.

We have already noted that the DCIS co-emptiness problem is decidable. The interreducibility claimed in Theorem 20 is proven in the rest of this section. We first define the set

$$
\mathcal{S}_{\mathcal{V}}=\{q(v) \mid \text { no } t \in T \text { is enabled in } q(v)\}
$$


of "sink configurations" or "deadlocks" (hence $\mathcal{S}_{\mathcal{V}}=\bigcap_{t \in T} \mathcal{D}_{t, \mathcal{V}}$ ). It is obvious that $\mathcal{S}_{\mathcal{V}}$ is the downward closed set described so that to each $q \in Q$ we attach the constraint

$$
\bigwedge_{\left(q \stackrel{\mathbf{a}}{\rightarrow} q^{\prime}\right) \in T} \bigvee_{\substack{i \in[1, d] \\ \mathbf{a}(i)<0}} v(i) \leq-\mathbf{a}(i)-1 .
$$

This presentation of $\mathcal{S}_{\mathcal{V}}$ can be clearly constructed in polynomial time, when given a VASS $\mathcal{V}$. Hence Proposition 21 entails the "left-to-right" reduction in Theorem 20 (recall that $\mathcal{V} \leftarrow$ denotes the reversed VASS of $\mathcal{V}$ ). The other reduction is shown by Proposition 22 .

Proposition 21. A VASS $\mathcal{V}$ is not structurally deadlock-free iff $\left[\mathcal{S}_{\mathcal{V}}\right\rangle_{\mathcal{V} \leftarrow}$ is coempty.

Proof. We consider a VASS $\mathcal{V}=(d, Q, \mathcal{A}, T)$, and observe that $q(v)$ is not deadlock-free iff $[q(v)\rangle_{\mathcal{V}} \cap \mathcal{S}_{\mathcal{V}} \neq \emptyset$. Hence $q(v)$ is not deadlock-free iff $q(v) \in$ $\left[\mathcal{S}_{\mathcal{V}}\right\rangle_{\mathcal{L}}$ (using Proposition 11). It follows that $\mathcal{V}$ is not structurally deadlockfree iff $\left[\mathcal{S}_{\mathcal{V}}\right\rangle_{\mathcal{V} \leftarrow}=Q \times \mathbb{N}^{d}$.

Proposition 22. Given a VASS $\mathcal{V}$ and a downward-closed set $D$ of configurations, we can construct, in polynomial time, a VASS $\mathcal{V}^{\prime}$ such that $[D\rangle_{\mathcal{V}}$ is co-empty iff $\mathcal{V}^{\prime}$ is not structurally deadlock-free.

Proof. Let us assume a VASS $\mathcal{V}=(d, Q, \mathcal{A}, T)$ and a downward-closed set $D$ of configurations given, for each $q \in Q$, by conjunctions of disjunctions of atomic constraints of the form $v(i) \leq c$. By negating these formulas, we derive, in polynomial time, a collection $\left(B_{q}\right)_{q \in Q}$ of finite subsets of $\mathbb{N}^{d}$ such that

$$
\left(Q \times \mathbb{N}^{d}\right) \backslash D=\left\{q(v) \mid v \geq b \text { for some } b \in B_{q}\right\} .
$$

(Hence $\left(B_{q}\right)_{q \in Q}$ represents the upward closed complement of $D$.)

We now define the VASS $\hat{\mathcal{V}}=(d, \hat{Q}, \hat{\mathcal{A}}, \hat{T})$ as follows:

a) $\hat{Q}=Q \cup\left\{(q, b) \mid q \in Q, b \in B_{q}\right\}$.

b) $\hat{T}$ consists of the following transitions:

i. $q \stackrel{-b}{\longrightarrow}(q, b)$ and $(q, b) \stackrel{b}{\rightarrow} q$ for all $q \in Q, b \in B_{q}$, and

ii. $(q, b) \stackrel{\mathbf{a}+b}{\longrightarrow} q^{\prime}$ for all $\left(q \stackrel{\mathbf{a}}{\rightarrow} q^{\prime}\right) \in T$ and $b \in B_{q}$.

c) $\hat{\mathcal{A}}=\left\{\hat{\mathbf{a}} \mid q \stackrel{\hat{a}}{\rightarrow} q^{\prime} \in \hat{T}\right.$ for some $\left.q, q^{\prime} \in \hat{Q}\right\}$.

It is obvious that for all configurations $q(v)$ and $q^{\prime}\left(v^{\prime}\right)$ of $\mathcal{V}$ we have that

$$
q(v) \stackrel{*}{\rightarrow}_{\hat{v}} q^{\prime}\left(v^{\prime}\right) \text { implies } q(v) \stackrel{*}{\rightarrow} \mathcal{v} q^{\prime}\left(v^{\prime}\right)
$$

but the converse does not hold in general. We will show that

$$
[D\rangle_{\mathcal{V} \leftarrow}=Q \times \mathbb{N}^{d} \text { iff } \hat{\mathcal{V}} \text { is not structurally deadlock-free. }
$$


The proof will be finished, by taking $\mathcal{V}^{\prime}=\widehat{\mathcal{V}^{\leftarrow}}$ (and noting that $\left.\left(\mathcal{V}^{\leftarrow}\right)^{\leftarrow}=\mathcal{V}\right)$.

$(\Rightarrow)$ Assume $[D\rangle_{\mathcal{V} \leftarrow}=Q \times \mathbb{N}^{d}$. Observe that $(q, b)(v) \stackrel{b}{\rightarrow} q(v+b)$ in $\hat{\mathcal{V}}$ for every $(q, b) \in \hat{Q}$ and $v \in \mathbb{N}^{d}$. We now show that no configuration $q(v)$ with $q \in Q$ is deadlock-free in $\hat{\mathcal{V}}$, which clearly entails that $\hat{\mathcal{V}}$ is not structurally deadlock-free.

Let us fix some $q(v) \in Q \times \mathbb{N}^{d}$. Since $q(v) \in[D\rangle_{\mathcal{V} \leftarrow}$, there are $\mathbf{a}_{1}, \ldots, \mathbf{a}_{m} \in \mathcal{A}$ and $q_{0}\left(v_{0}\right), \ldots, q_{m}\left(v_{m}\right) \in\left(Q \times \mathbb{N}^{d}\right)$ such that

$$
q(v)=q_{0}\left(v_{0}\right) \stackrel{\mathbf{a}_{1}}{\longrightarrow} q_{1}\left(v_{1}\right) \stackrel{\mathbf{a}_{2}}{\longrightarrow} \cdots q_{m-1}\left(v_{m-1}\right) \stackrel{\mathbf{a}_{m}}{\longrightarrow} q_{m}\left(v_{m}\right) \in D \text { in } \mathcal{V}
$$

(recall Proposition 11). Moreover, we may assume w.l.o.g. that $q_{i}\left(v_{i}\right) \notin D$ for all $i \in[0, m-1]$. So for each $i \in[0, m-1]$ there is $b_{i} \in B_{q_{i}}$ such that $v_{i} \geq b_{i}$. We derive that

$$
q_{i}\left(v_{i}\right) \stackrel{-b_{i}}{\longrightarrow}\left(q_{i}, b_{i}\right)\left(v_{i}-b_{i}\right) \stackrel{\mathbf{a}_{i+1}+b_{i}}{\longrightarrow} q_{i+1}\left(v_{i+1}\right) \text { in } \hat{\mathcal{V}}
$$

for all $i \in[0, m-1]$. It follows that $q(v) \stackrel{*}{\rightarrow} q_{m}\left(v_{m}\right)$ in $\hat{\mathcal{V}}$. Since $q_{m}\left(v_{m}\right) \in D$ then $v_{m} \nsupseteq b$ for all $b \in B_{q_{m}}$; hence no transition of $\hat{\mathcal{V}}$ is enabled in $q_{m}\left(v_{m}\right)$, and $q(v)$ is thus not deadlock-free in $\hat{\mathcal{V}}$.

$(\Leftarrow)$ Assume that $\hat{\mathcal{V}}$ is not structurally deadlock-free. We fix a configuration $q(v)$ of $\mathcal{V}$ and prove that $q(v) \in[D\rangle_{\mathcal{V} \leftarrow}$. Since $q(v)$ is also a configuration of $\hat{\mathcal{V}}$, it is not deadlock-free in $\hat{\mathcal{V}}$. So there is a configuration $q^{\prime}\left(v^{\prime}\right)$ of $\hat{\mathcal{V}}$ such that $q(v) \stackrel{*}{\rightarrow}_{\hat{\mathcal{V}}} q^{\prime}\left(v^{\prime}\right)$ and no transition $t \in \hat{T}$ is enabled in $q^{\prime}\left(v^{\prime}\right)$. Since $\hat{\mathcal{V}}$ contains the transition $(q, b) \stackrel{b}{\rightarrow} q$ for every $q \in Q$ and $b \in B_{q}$, we get that $q^{\prime} \in Q$. No transition $q^{\prime} \stackrel{-b}{\longrightarrow}\left(q^{\prime}, b\right)$ of $\hat{T}$ is enabled in $q^{\prime}\left(v^{\prime}\right)$, so $v^{\prime} \nsucceq b$ for every $b \in B_{q^{\prime}}$. It follows that $q^{\prime}\left(v^{\prime}\right) \in D$. Since $q(v) \stackrel{*}{\rightarrow} \hat{\mathcal{v}} q^{\prime}\left(v^{\prime}\right)$ implies $q(v) \stackrel{*}{\rightarrow} \mathcal{v} q^{\prime}\left(v^{\prime}\right)$, we get $q(v) \stackrel{*}{\rightarrow} \mathcal{V} D$, i.e., $q(v) \in[D\rangle_{\mathcal{V} \leftarrow}$.

\section{Conclusion}

Motivated by the structural liveness problem for VASS, whose computational complexity is still open, we have introduced and studied in this paper the coemptiness problem and the co-finiteness problem for VASS. The complexity of the co-emptiness and co-finiteness problems in the case of single initial configurations has been clarified, but the complexity of general versions has been left open, even w.r.t. reductions to/from the reachability problem. This requires further work, in particular with an eye to the applications aiming to clarify structural liveness properties of VASSs, or equivalently of Petri nets.

\section{References}

1. Atig, M.F., Habermehl, P.: On Yen's path logic for Petri nets. Int. J. Found. Comput. Sci. 22(4), 783-799 (2011), https://doi.org/10.1142/S0129054111008428 
2. Best, E., Esparza, J.: Existence of home states in Petri nets is decidable. Inf. Process. Lett. 116(6), 423-427 (2016)

3. Esparza, J.: Decidability and complexity of Petri net problems - an introduction. In: Advances in Petri Nets '98. LNCS, vol. 1491, pp. 374-428. Springer (1998)

4. Ginsburg, S., Spanier, E.H.: Semigroups, Presburger formulas, and languages. Pacific J. Math. 16(2), 285-296 (1966)

5. Hack, M.: The recursive equivalence of the reachability problem and the liveness problem for Petri nets and vector addition systems. In: 15th Annual Symposium on Switching and Automata Theory, New Orleans, Louisiana, USA, October 14-16, 1974. pp. 156-164. IEEE Computer Society (1974), http://dx.doi.org/10.1109/SWAT.1974.28

6. Hopcroft, J.E., Pansiot, J.: On the reachability problem for 5-dimensional vector addition systems. Theor. Comput. Sci. 8, 135-159 (1979)

7. Jančar, P.: Deciding structural liveness of Petri nets. In: SOFSEM 2017. Lecture Notes in Computer Science, vol. 10139, pp. 91-102. Springer (2017)

8. Leroux, J.: Presburger vector addition systems. In: 28th Annual ACM/IEEE Symposium on Logic in Computer Science, LICS 2013, New Orleans, LA, USA, June 25-28, 2013. pp. 23-32. IEEE Computer Society (2013), http://dx.doi.org/10.1109/LICS.2013.7

9. Leroux, J.: Vector addition system reversible reachability problem. Logical Methods in Computer Science 9(1) (2013), https://doi.org/10.2168/LMCS-9(1:5)2013

10. Leroux, J., Schmitz, S.: Demystifying reachability in vector addition systems. In: 30th Annual ACM/IEEE Symposium on Logic in Computer Science, LICS 2015, Kyoto, Japan, July 6-10, 2015. pp. 56-67. IEEE Computer Society (2015), http://dx.doi.org/10.1109/LICS.2015.16

11. Lipton, R.J.: The reachability problem requires exponential space. Tech. Rep. 63, Department of Computer Science, Yale University (Jan 1976)

12. Mayr, E.W.: An algorithm for the general Petri net reachability problem. SIAM J. Comput. 13(3), 441-460 (1984), http://dx.doi.org/10.1137/0213029

13. Rackoff, C.: The covering and boundedness problems for vector addition systems. Theor. Comput. Sci. 6, 223-231 (1978), http://dx.doi.org/10.1016/03043975(78)90036-1

14. Yen, H.: A unified approach for deciding the existence of certain Petri net paths. Inf. Comput. 96(1), 119-137 (1992), https://doi.org/10.1016/0890-5401(92)90059$\mathrm{O}$ 